\title{
Microfluidic model of bubble lodging in microvessel bifurcations
}

\author{
Andrés J. Calderón, Yun Seok Heo, Dongeun Huh, Nobuyuki Futai, and Shuichi Takayama \\ Department of Biomedical Engineering, University of Michigan, Ann Arbor, Michigan 48109 \\ J. Brian Fowlkes \\ Department of Radiology, University of Michigan, Ann Arbor, Michigan 48109 \\ Joseph L. Bull ${ }^{\text {a) }}$ \\ Department of Biomedical Engineering, University of Michigan, Ann Arbor, Michigan 48109
}

(Received 12 September 2006; accepted 26 October 2006; published online 12 December 2006)

\begin{abstract}
The lodging mechanisms and dynamics of cardiovascular gas bubbles are investigated in microfluidic model bifurcations made of poly(dimethylsiloxane). This work is motivated by gas embolotherapy for the potential treatment of cancer by tumor infarction. The results show that the critical driving pressure below which a bubble will lodge in a bifurcation is significantly less than the driving pressure required to dislodge it. From the results the authors estimate that gas bubbles from embolotherapy can lodge in vessels $20 \mu \mathrm{m}$ or smaller in diameter, and conclude that bubbles may potentially be used to reduce blood flow to tumor microcirculation. (c) 2006 American Institute of Physics. [DOI: 10.1063/1.2402898]
\end{abstract}

Lodging of cardiovascular gas bubbles in single and multiple arteriole bifurcations is investigated in a microfluidic model. Our investigation is primarily motivated by developmental gas embolotherapy aimed at selective infarction of tumors, but has other applications such as air embolism and bubble lodging in microfluidic devices. ${ }^{1}$ Gas embolotherapy uses albumin-encapsulated perfluorocarbon (PFC) droplets that can be monitored and selectively vaporized with ultrasound to form gas bubbles that are sufficiently large to occlude blood flow when lodged in the tumor microcirculation. ${ }^{1,2}$ Understanding the dynamics of bubble lodging is essential for knowing where to vaporize the PFC droplets to infarct the targeted vessel. Bubble lodging is a complex phenomena and is difficult to study systematically with in vivo studies. Although actual vessels have an endothelial glycocalyx layer, ${ }^{3}$ we note that a number of studies have identified and investigated the dominant mechanisms of leukocyte adhesion in simplified systems. ${ }^{4}$ Therefore, we investigated bubble lodging in a microfluidic model so that we are able to vary multiple factors, such as bubble size, bifurcating angle, parent to daughter channel ratios, and cross sectional shape and area.

Model bifurcations were patterned on poly(dimethylsiloxane) (PDMS) by replica molding and then sealed against a slab of PDMS using a plasma oxidizer (11005-Plasma Prep II, SPI, West Chester, PA). ${ }^{5}$ Rounded and smooth channel sidewalls were generated by using backside diffused-light photolithography (Fig. 1). ${ }^{6}$ We also fabricated channels of square cross sections by conventional photolithography. ${ }^{5} \mathrm{~A}$ single air bubble was injected in water within the parent channel of the bifurcation using a $10 \mu$ l syringe (Hamilton; Reno, NV) and a 26 gauge needle (Hamilton 7758-04). This was repeated for several dimensionless bubble lengths, $L_{d}$, which corresponds to the length of the bubble divided by the hydraulic diameter (HD). A specified driving pressure, $\Delta P$, was imposed to move the bubble by adjusting the reservoir elevations. We measured the $\Delta P$ at which bubbles lodged and subsequently dislodged in the bifurcation by measuring the difference in reservoir elevations. Figure 1(d) shows

\footnotetext{
${ }^{a)}$ Electronic mail: joebull@umich.edu
}

the dimensions for the different single bifurcations investigated here, the angle between the daughter channels (bifurcation angle), perimeter ratio, and HD ratio. The angles and HD ratios of these bifurcations were chosen to approxi-

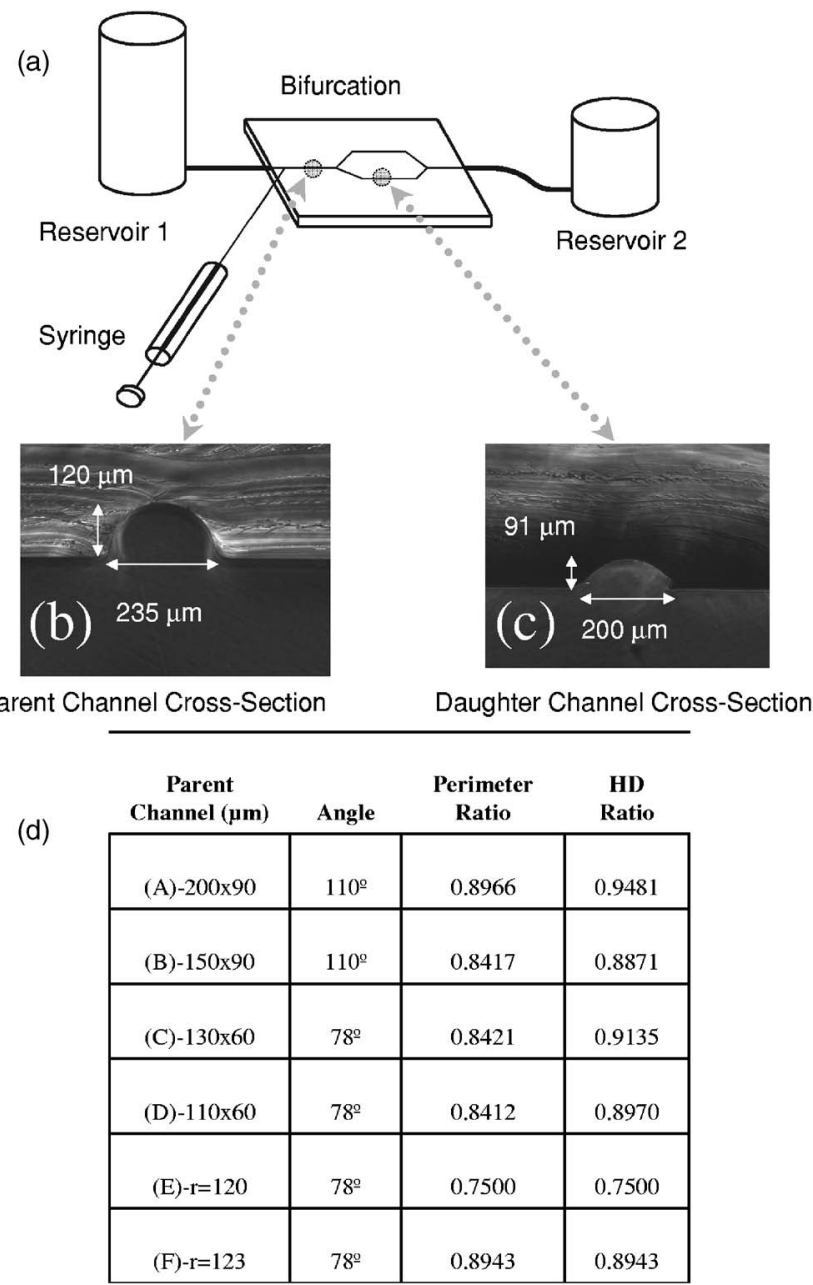

FIG. 1. (a) Schematic of the experimental setup. Cross sections of rounded (b) parent and (c) daughter channels. (d) Bifurcation dimensions and geometry. $r=$ radius. 

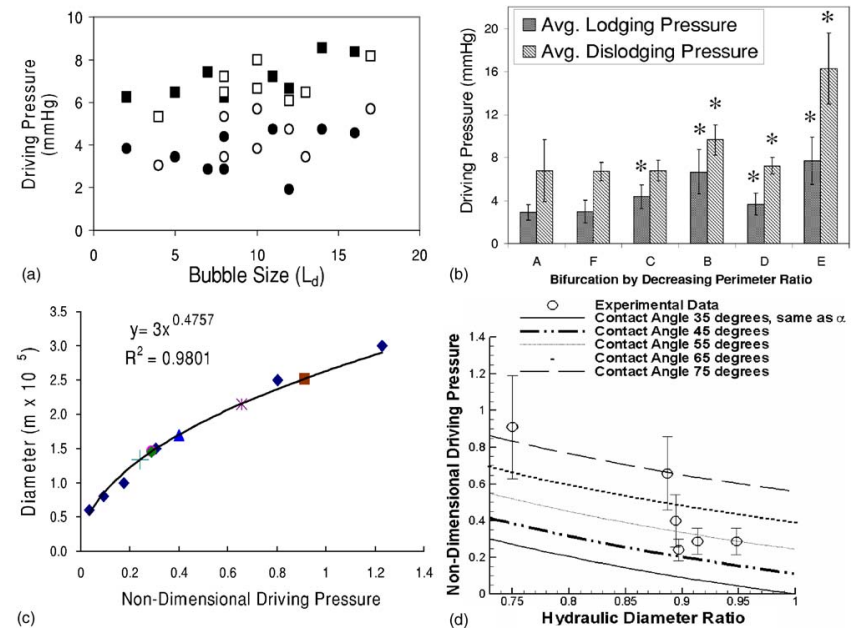

FIG. 2. (Color online) (a) $\Delta P$ vs $L_{d}$ for bubble lodging in $150 \mu \mathrm{m}$ ( ) and $200 \mu \mathrm{m}(\bigcirc)$ channels, and dislodging in $150 \mu \mathrm{m}(\square)$ and $200 \mu \mathrm{m}(\square)$ channels. (b) Lodging and dislodging $\Delta P$ for all the bifurcations, ${ }^{*}$ indicates $p<0.05$. (c) Nondimensional physiologic $\Delta P$ vs vessel diameter. The data points correspond to the nondimensional lodging $\Delta P$ for each bifurcation. (d) Theoretical curves and experimental data, HD ratio vs nondimensional $\Delta P$.

mate normal physiologic values ${ }^{7}$ as an initial model. However, the microcirculation of actual tumors is chaotic and axisymmetric.

In all of the bifurcations the critical pressure for lodging was smaller than the dislodging pressure, see Fig. 2(a). This difference in pressure is due to contact angle (CA) hysteresis. ${ }^{8,9}$ These results suggest that once bubbles lodge in the microcirculation of tumors, higher $\Delta P$ are needed to dislodge them. Bubble length did not change lodging and dislodging pressures significantly. There were two states in which bubbles lodged at the bifurcation: lodging states $\mathrm{A}$ and B [Figs. 3(a) and 3(b), respectively]. Bubbles usually lodged in state A, where the bubble's front and back menisci contacted the wall. Most of the bubbles lodged in this state originally, and as the pressure was increased, the bubble either dislodged or lodged again at a higher pressure in state B. In Fig. 3(b) the rear meniscus appears to stretch and eventually prevents the bubble from moving. Lodging state B was more common in the microchannels with the bifurcation angle of

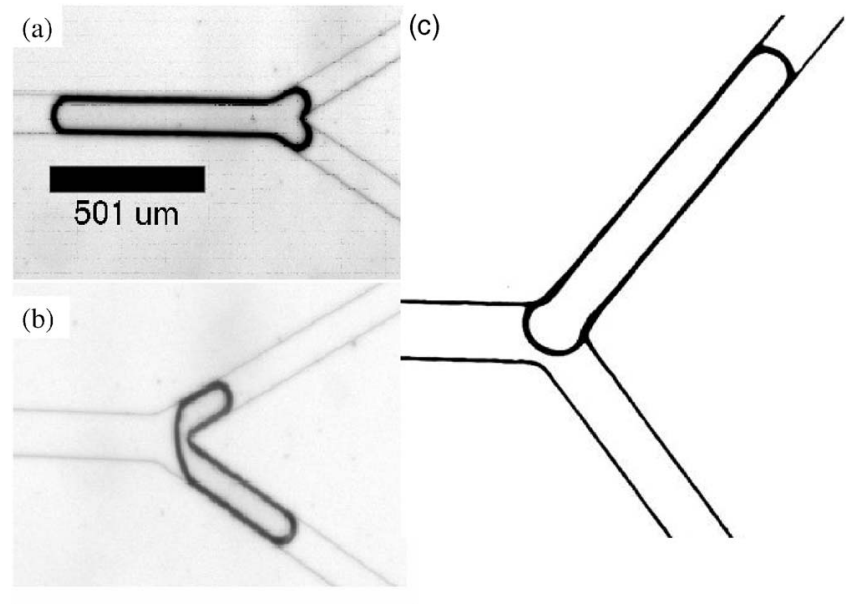

FIG. 3. [(a) and (b)] Lodging states A and B. (c) Bubble lodged in one branch after it reversed from the bottom branch. $10 \times$ magnification.
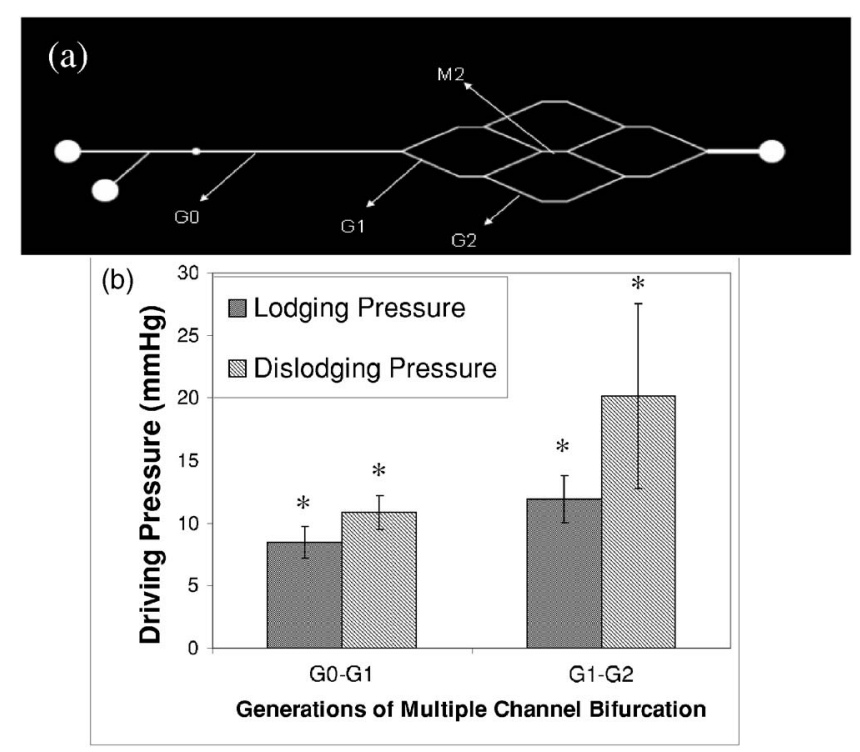

FIG. 4. (a) Schematic of multiple channel bifurcation. The perimeter ratios for the first and second bifurcations are $\approx 0.80$ and 0.71 , respectively. (b) Average lodging and dislodging $\Delta P$ for the multiple bifurcation, ${ }^{*}$ indicates $p<0.05$.

$110^{\circ}$, in which the lodged bubble could withstand higher $\Delta P$ than in the $78^{\circ}$ bifurcation.

We observed bubble reversal similar to that in our previous studies of bubble transport, ${ }^{10}$ where the bubble begins to split and then one of the menisci returns and the bubble does not split and travels into one branch. In some instances when the $\Delta P$ was increased to dislodge the bubble, the shorter portion of the bubble in the daughter branch returned and the rear meniscus bulged such that the bubble lodged in just one branch [Fig. 3(c)]. In this case no matter how much we increased the pressure $(\approx 55 \mathrm{mmHg}$ ) the bubble did not dislodge, and the flow was shunted to the unoccluded branch. This was verified by observing the transport of $10 \mu \mathrm{m}$ diameter microspheres (Dantec Dynamics HGS-10, Skovlunde, Denmark), which all went to the unoccluded branch. We could not observe any clear set of conditions for bubble reversal.

The perimeter ratios (parent channel: daughter channel) were very similar to one another, but lower perimeter ratios generally resulted in higher lodging and dislodging $\Delta P$, as shown in Fig. 2(b) $(p<0.05$ from analysis of variance was considered significant). We investigated the homogeneity of bubble splitting and noted an instability region where bubbles did not split evenly. This instability occurs with $\Delta P$ in between the lodging and dislodging pressures. Increasing the $\Delta P$ above the dislodging pressure reduced the instability. We attribute this instability to bubbles contacting the wall at lower pressures, thus exhibiting a "stick and slip" behavior. ${ }^{8}$ At pressures higher than dislodging pressures, the bubbles usually had a lubricating film between the bubble and the wall, ${ }^{11}$ which allows for higher splitting homogeneity due to the lack of "stick and slip" behavior.

A bifurcating network consisting of multiple bifurcations was also studied to understand bubble shunting and lodging in a circuit of bifurcations, see Fig. 4(a). The same type of lodging states occurred in the multiple bifurcations. In the multiple bifurcations it was more common to observe bubbles lodged in the daughter channels of the bifurcation as opposed to the bifurcation itself. In all the runs at least one 
bubble lodged in a straight channel. The pressures to lodge and dislodge the bubbles in the multiple channel bifurcations were higher than the single bifurcation experiments. Figure 4(b) shows the lodging and dislodging $\Delta P$ for the first and second generation bifurcations. The increase in dislodging $\Delta P$ (increased by up to $46 \%$ ) was more significant than the increase in lodging $\Delta P$ (increased up to $12.5 \%$, compared to the single bifurcation of perimeter ratio of 0.75 ).

We also introduced additional bubbles after the initial bubble lodged in the second generation bifurcations or in additional second generation daughter branches to determine if the bubbles can occlude the regions where flow was shunted. We were able to occlude the entire circuit using several bubbles, at the highest lodging pressure. The $\Delta P$ needed to dislodge all the bubbles was higher than the physiologic values. Microspheres introduced into the fluid were stationary in the occluded regions and moving in the shunted areas, suggesting that the lodged bubbles occluded the channel or bifurcations. In some cases microspheres appeared to move in the occluded regions, but at very slow speeds, approximately $1-5 \mu \mathrm{m} / \mathrm{s}$, suggesting that there was a small gap $(<10 \mu \mathrm{m})$ between the bubble interface and the wall. Based on these observations, we expect that as bubbles lodge in the microcirculation of tumors, subsequent bubbles will occlude the shunted areas of flow, resulting in almost complete occlusion of the tumor.

To estimate the pressure at which bubbles might lodge in the vasculature we calculated the differential pressure at various levels of the vasculature and plotted vessel diameter versus nondimensional pressure, see Fig. 2(c). Using a power regression we calculated which size of vessel corresponds to the experimental nondimensional pressures. Based on the experimental results, we anticipate that some bubbles will lodge in $20 \mu \mathrm{m}$ diameter vessels and that any remaining bubbles will lodge by the time they reach $15-10 \mu \mathrm{m}$ diameter vessels. At that location, the bubbles will be sausage shaped $\left(L_{d}>2\right)$ rather than spherical and the $\Delta P$ is below the anticipated lodging pressure, see Fig. 2(c). In order to elucidate the mechanisms involved and predict bubble lodging behavior, we developed a theoretical model of a static bubble lodged in a bifurcation. Applying Laplace's law ${ }^{12}$ to each interface of the bubble and combining the resulting expressions yield an expression for $\Delta P$ that can be supported by the bubble. We nondimensionalized the equations by $\hat{D}$ $=h d / h D$ and $\hat{P}=\Delta P h D /(4 \sigma)$, where $\sigma$ is the surface tension, and $h D$ and $h d$ are the hydraulic diameters of the parent and daughter tubes, respectively. The $\mathrm{CA}$ in the parent and daughter tubes are $\beta$ and $\alpha$, respectively. The resulting dimensionless equation for the pressure that can be supported by a lodged bubble is $\hat{P}=(1 / \hat{D}-\cos \alpha / \cos \beta) \cos \alpha$. Figure 2(d) is truncated to fit only a physiological range of $\hat{D}$ and of CA. The graph also includes the experimental values of all the single bifurcations using the HD ratio as the diameter ratio for square bifurcations. The experimental values for nondimensional pressure are in good agreement with the theoretical model for the values of $\alpha$ and $\beta$ measured from the experimental work. We use the same $\mathrm{CA}, \alpha$, in both daughter tubes because we did not observe a significant difference between the front CAs, but the rear CA was observed to vary depending on how the bubble lodged. From the theory we can infer that increasing the difference between the rear CA and the front CAs increases the critical $\Delta P$ for lodging. This critical $\Delta P$ for lodging is decreased by increasing the parent to daughter HD ratio [Fig. 2(d)]. Based on the theoretical analysis and typical physiologic pressures and diameter ratios, $^{7}$ we estimate that bubbles may begin to lodge in a parent vessel of size $28 \mu \mathrm{m}$ with daughter vessels of $21.8 \mu \mathrm{m}$. The critical pressures in these experiments are higher than the anticipated pressure drop across small arteriole bifurcations, in the range of $20-15 \mu \mathrm{m}$, suggesting that gas emboli might begin to lodge there, where $L_{d} \approx 2$.

Although not considered here, the glycocalyx of the endothelium might influence bubble lodging through interactions with charged surface-active species that adsorb on the bubble interface and through fluid mechanical lubrication. In studies of adhesion of long bubbles in straight excised arterioles, the adhesive force on lodged bubbles was of the same order, but different value, for vessels with and without an intact endothelium, ${ }^{13}$ suggesting that this study provides reasonable estimates of the location and state of microbubble lodging.

In summary, this study provides insights into bubble lodging in microvessel bifurcations, and indicates the potential of using PFC bubbles for gas embolotherapy. Dislodging pressures were shown to be significantly higher (approximately double) than lodging pressures, suggesting that once lodged, bubbles tend to remain lodged. Based on the experimental and theoretical results, we expect microbubbles in gas embolotherapy to lodge in microvessels with diameters of $20 \mu \mathrm{m}$ and less. Lodged bubbles occluded the flow of microspheres, suggesting that red blood cells will not be transported to the tumor, causing tumor necrosis, consistent with the goal of gas embolotherapy. The inverse relationship between dimensionless lodging pressure and surface tension suggests that reducing surface tension, perhaps through surfactants, is a possible way to prevent bubble lodging or to dislodge lodged bubbles. The latter may be particularly relevant to treatment of air embolism and to two-phase microfluidic circuits, in which lodged bubbles are unwanted.

This work was funded by NIH (EB003541 and HL084370) and NSF (BES-0301278).

${ }^{1}$ J. L. Bull, Crit. Rev. Biomed. Eng. 33, 299 (2005).

${ }^{2}$ O. D. Kripfgans, J. B. Fowlkes, D. L. Miller, O. P. Eldevik, and P. L. Carson, Ultrasound Med. Biol. 26, 1177 (2000).

${ }^{3}$ E. R. Damiano and T. M. Stace, Phys. Fluids 17, 031509 (2005); T. W. Secomb, R. Hsu, and A. R. Pries, Am. J. Physiol. Heart Circ. Physiol. 281, H629 (2001).

${ }^{4}$ D. K. Brunk, D. J. Goetz, and D. A. Hammer, Biophys. J. 71, 2902 (1996); D. A. Hammer and S. M. Apte, ibid. 63, 35 (1992).

${ }^{5}$ D. C. Duffy, J. C. McDonald, O. J. A. Schueller, and G. M. Whitesides, Anal. Chem. 70, 4974 (1998).

${ }^{6}$ N. Futai, W. Gu, and S. Takayama, Adv. Mater. (Weinheim, Ger.) 16, 1320 (2004).

${ }^{7}$ Y. C. Fung, Biomechanics: Circulation (Springer, New York, 1997), p. 571.

${ }^{8}$ D. M. Eckmann, D. P. Cavanagh, and A. B. Branger, J. Colloid Interface Sci. 242, 386 (2001).

${ }^{9}$ C. W. Extrand and Y. Kumagai, J. Colloid Interface Sci. 191, 378 (1997).

${ }^{10}$ A. J. Calderon, J. B. Fowlkes, and J. L. Bull, J. Appl. Physiol. 99, 479 (2005).

${ }^{11}$ F. P. Bretherton, J. Fluid Mech. 10, 166 (1961); T. Cubaud and C.M. Ho, Phys. Fluids 16, 4575 (2004).

${ }^{12}$ H. K. Chang, M. E. Weber, J. Thomson, and R. R. Martin, J. Appl. Physiol. 51, 1002 (1981).

${ }^{13}$ A. Suzuki and D. M. Eckmann, Anesthesiology 99, 400 (2003). 